\title{
Motivational states influence effort-based decision making in rats: The role of dopamine in the nucleus accumbens
}

\author{
Bettina Mai • Susanne Sommer • Wolfgang Hauber
}

Published online: 20 October 2011

(C) Psychonomic Society, Inc. 2011

\begin{abstract}
Decision-making policies are subject to modulation by changing motivational states. However, so far, little is known about the neurochemical mechanisms that bridge motivational states with decision making. Here we examined whether dopamine (DA) in the nucleus accumbens core $(\mathrm{AcbC})$ modulates the effects of motivational states on effort-based decision making. Using a cost-benefit T-maze task in rats, we examined the effects of AcbC DA depletions on effort-based decision making, in particular on the sensitivity of effort-based decision making to a shift from a hungry to a sated state. The results demonstrated that, relative to sham controls, rats with AcbC DA depletion in a hungry as well as in a sated state had a reduced preference for effortful but large-reward action. This finding provides further support for the notion that AcbC DA regulates how much effort to invest for rewards. Importantly, our results further revealed that effort-based decision making in lesioned rats, as in sham controls, was still sensitive to a shift from a hungry to a sated state; that is, their preferences for effortful large-reward actions became lower after a shift from a restricted to a freefeeding regimen. These finding indicate that AcbC DA is not necessarily involved in mediating the effects of a shift in motivational state on decision-making policies.
\end{abstract}

Keywords 6-Hydroxydopamine $\cdot$ Rat $\cdot$ Nucleus accumbens $\cdot$ Effort-based decision making $\cdot$ Motivational states

\footnotetext{
B. Mai $\cdot$ S. Sommer $\cdot$ W. Hauber $(\triangle)$

Biologisches Institut, Abteilung Tierphysiologie,

Universität Stuttgart,

Pfaffenwaldring 57,

D-70550 Stuttgart, Germany

e-mail: hauber@bio.uni-stuttgart.de
}

Motivational states play a crucial role in decision making and the implementation of behavioural strategies. For instance, in an iterated prisoner's dilemma game for rats, social interaction that leads to reward but entails costs to the interacting individuals was lower in hungry than in sated individuals, highlighting a role for motivational states in reciprocity-based cooperation (Viana, Gordo, Sucena, \& Moita, 2010). Furthermore, for rats tested in effort-based decision-making tasks, a shift in motivational state by means of satiety manipulations changed their preference from high-value-high-cost options to low-value-low-cost alternatives (Endepols, Sommer, Backes, Wiedermann, Graf \& Hauber, 2010; Floresco, Tse, \& Ghods-Sharifi, 2008; Salamone, Steinpreis, McCullough, Smith, Grebel \& Mahan, 1991). While these findings suggest that decisionmaking policies are variable and subject to modulation by changing motivational states, little is known about the neurochemical mechanisms that bridge motivational states such as hunger or thirst with decision making. In rats examined in feeding tasks, microdialysis studies revealed that dopamine (DA) release in the nucleus accumbens is modulated by motivational state (Ahn \& Phillips, 1999; Bassareo \& Di Chiara, 1999; Wilson, Nomikos, Collu, \& Fibiger, 1995). On the basis of these findings, changes in accumbens DA efflux have been suggested to mediate effects of motivational states on effort-based decision making (Phillips, Walton, \& Jhou, 2007); however, empirical evidence in support of this account is lacking. Recent studies have further indicated that the core subregion of the nucleus accumbens (AcbC; Hauber \& Sommer, 2009; Salamone, Correa, Farrar, \& Mingote, 2007), but not the shell subregion (AcbS; Ghods-Sharifi \& Floresco, 2010), plays a key role in effort-based decision making. Moreover, accumbens DA depletions encompassing AcbC and AcbS rendered rats cost-aversive (Cousins, Atherton, Turner, \& 
Salamone, 1996; Salamone, Cousins, \& Bucher, 1994). Thus, it is conceivable that accumbens DA plays a dual role in effort-based decision making, biasing responding to high-value-high-cost options and mediating the effects of motivational states.

Here we sought to explore the role of AcbC DA in mediating the effects of motivational states on effort-based decision making. Using a T-maze cost-benefit task, we examined in Experiment 1 whether effort-based decision making in intact rats was sensitive to a shift from a hungry to a sated state. In this task, subjects could either choose to climb a barrier $(30 \mathrm{~cm})$ to obtain a high reward $(\mathrm{HR}=4$ pellets) in one arm, or to obtain a low reward (LR $=2$ pellets) in the other arm without a barrier (Cousins et al., 1996; Salamone et al., 1994; Schweimer \& Hauber, 2005; Walton, Bannerman, Alterescu, \& Rushworth, 2003). In Experiment 2, we examined the effects of AcbC DA depletions on effort-based decision making, in particular on the sensitivity of effort-based decision making to a shift from a hungry to a sated state. As mesoaccumbens DA is critical for enabling an organism to overcome response costs to gain access to greater reward (Cousins \& Salamone, 1994; Salamone, 1994; Salamone, Correa, Farrar \& Mingote, 2007), rats with AcbC DA depletion should have a reduced preference for effortful but large-reward action both in a hungry and in a sated state. Furthermore, if DA also conveys signals related to motivational state, as has been suggested by earlier studies (Wilson et al., 1995), effort-based decision making in rats with AcbC DA depletion should be insensitive to a shift from a hungry to a sated state.

\section{Method}

Experiments were performed according to the German Law on Animal Protection and were approved by the proper authorities.

\section{Experiment 1}

In Experiment 1, we examined whether effort-based decision making in intact rats was sensitive to a shift in motivational state - that is, from a hungry to a sated state.

Subjects Male Sprague-Dawley rats (Charles River, Sulzfeld, Germany) were housed in groups of up to 4 animals in transparent macrolon cages (type IV; $35 \times 55 \times 10 \mathrm{~cm}$; Ebeco, Castrop-Rauxel, Germany) in a 12:12-h light: dark cycle (lights on at 7:00 AM) with ad libitum access to water. During habituation and training, lab chow (standard maintenance chow; Altromin, Lage, Germany) was limited to $15 \mathrm{~g}$ per animal per day. Subsequently, effort-based decision making was examined under either a restricted feeding regimen ( $12 \mathrm{~g}$ of chow per animal per day) or a free-feeding regimen (ad libitum access to chow). Under restriction conditions, the appropriate amount of lab chow was delivered to the food well of each cage in the afternoon. For environmental enrichment, a plastic tube $(20 \mathrm{~cm}, \varnothing 12 \mathrm{~cm})$ was fixed on the lid of each cage. Temperature $\left(22 \pm 2^{\circ} \mathrm{C}\right)$ and humidity $(50 \% \pm 10 \%)$ were kept constant in the animal house.

Apparatus A T-maze task involving effort-based decision making was used (e.g., Schweimer \& Hauber, 2005). The elevated T-maze consisted of a start and two goal arms (17 cm wide, $68 \mathrm{~cm}$ long) made of laminated wood; the walls were $30 \mathrm{~cm}$ high. A food well was placed at the end of each goal arm. On forced trials, a solid block was used to prevent the animal from entering one goal arm. The barriers that the animal had to surmount were made of wire mesh in shape of a right-angled triangle. The rats had to climb the vertical side of the triangle and to descend the slope to attain the reward. The height of the barriers was increased during training from $15 \mathrm{~cm}$ to a final height of $30 \mathrm{~cm}$.

Habituation Rats were habituated to handling for 2 days. Then they were habituated to the T-maze on two subsequent days. On these days, the rats were placed in groups in the start arm and were allowed to explore the maze for $10 \mathrm{~min}$. Both goal arms contained casein pellets (45-mg dustless precision pellets; Bioserv, Frenchtown, NJ).

Training and test procedure After habituation to the maze, animals learned to discriminate the HR goal arm (= 4 food pellets) from the LR goal arm (= 2 food pellets). For one half of the group, the HR arm was on the right, and for the other half it was on the left. On Day 1, animals received 7 trials, in which they could explore both goal arms (see Fig. 1 for a time line). On Days 2-3, animals received 10 forced trials per day in which they were forced to run in opposite directions on subsequent trials. On Days 4-6, 12 trials were given per day; the first 2 trials of each day were forced trials, followed by 10 choice trials. On Day 6, HR preference in choice trials was $\geq 80 \%$. On Days $7-9$, a 15 -cm barrier was introduced into the HR arm. On Day 9, animals selected the HR arm in the majority of trials ( $\geq 70 \%)$. On Days $10-12$, the height of the barrier was increased to $20 \mathrm{~cm}$. On Days 13-15, the animals were trained with a barrier height of $25 \mathrm{~cm}$. Thereafter, Experiment 1 started.

Experiment 1 consisted of two subsequent testing blocks of 3 days each. In Testing Block 1, effort-based decision making was examined under a restrictive feeding regimen, and in Testing Block 2, under an ad libitum feeding regimen. Upon completion of behavioral testing on Day 3 


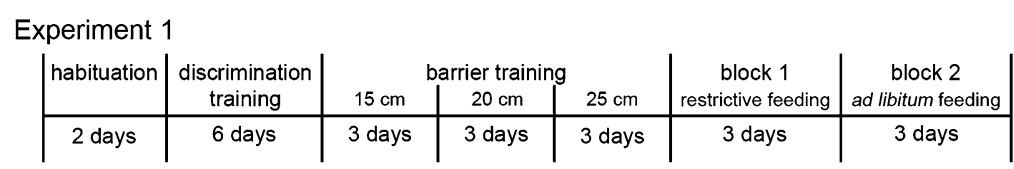

\begin{tabular}{|c|c|c|c|c|c|c|c|c|c|}
\hline \multicolumn{10}{|c|}{ Experiment 2} \\
\hline habituation & surgery \& & discrimination & \multicolumn{3}{|c|}{ barrier training } & block 1 & block 2 & bloc & consur \\
\hline & recovery & training & $15 \mathrm{~cm}$ & $20 \mathrm{~cm}$ & $25 \mathrm{~cm}$ & restrictive feeding & ad libitum feeding & restrictive feeding & restrictive feeding \\
\hline 2 days & 12 days & 6 days & 3 days & 3 days & 3 days & 5 days & 5 days & 3 days & 1 day \\
\hline
\end{tabular}

Fig. 1 Time lines with the behavioral manipulations in Experiments 1 and 2

of Testing Block 1, the feeding regimen was shifted, and rats had ad libitum access to food. Testing on Day 1 of Block 2 started $18 \mathrm{~h}$ after ad libitum access to food had begun. In this condition, a cost-benefit task that included one barrier ("one-barrier condition"), rats could choose either to mount a barrier for HR in one goal arm or to obtain LR in the other goal arm without a barrier; that is, an integration of the effort (climbing vs. not climbing) and the reward magnitudes (high vs. low) of the available response options was required before making a decision.

All rats completed every trial in all testing blocks. From all testing blocks, percentages of high-reward (\%HR) choices, response times (RTs, defined as the latency from insertion of an animal into the start arm until arrival at the reward position in a goal arm), and daily body weight were recorded.

Data analysis and statistics Choices of the HR arm, RTs, and body weights are given as percentage means \pm the standard error of each mean (SEM). Data were subjected to a repeated measures ANOVA with two within-subjects factors (days of testing, testing blocks). All statistical computations were carried out with STATISTICA (Version 7.1; StatSoft, Inc., Tulsa, OK). The level of statistical significance ( $\alpha$ level) was set at $p \leq .05$.

\section{Experiment 2}

In Experiment 2, we examined whether effort-based decision making in rats subjected to AcbC DA depletion was sensitive to a shift in motivational state - that is, a shift from a hungry to a sated state.

Subjects Male Sprague-Dawley rats (Charles River, Sulzfeld, Germany) ( $n=30)$ were maintained as described for Experiment 1.

Habituation Rats were habituated to handling for 2 days. Then, they were habituated to the T-maze on two subsequent days. On these days, rats were placed in groups in the start arm and were allowed to explore the maze for
30 min. Both goal arms contained casein pellets (45 mg dustless precision pellets; Bioserv, Frenchtown, NJ). Thereafter, animals were subjected to stereotaxic surgery.

Surgery After pretreatment with atropine sulfate $(0.5 \mathrm{mg} / \mathrm{kg}$, subcutaneously; WDT, Garbsen, Germany), the animals were anesthetized with sodium pentobarbital $(60 \mathrm{mg} / \mathrm{kg}$, intraperitoneally; Narcoren, Merial, Hallbergmoos, Germany) and xylazine (4 mg/kg, intramuscularly; Rompun, Bayer, Leverkusen, Germany) and secured in a stereotaxic apparatus with atraumatic ear bars (Kopf Instruments, Tujunga, USA). The skull was exposed and two small holes were drilled bilaterally above the AcbC core. Lesions were made by infusions of $4 \mu \mathrm{g}$ 6-hydroxydopamine (6OHDA) hydrochlorid (Sigma-Aldrich, Steinheim, Germany) in $0.4 \mu \mathrm{l}$ saline containing $0.01 \%$ ascorbic acid (SigmaAldrich, Steinheim, Germany) at the following coordinates using a 1- $\mu$ l Hamilton syringe: AP $+1.2 \mathrm{~mm}, \mathrm{ML} \pm 2.1 \mathrm{~mm}$, DV $-7.0 \mathrm{~mm}$, with the tooth bar at $-3.3 \mathrm{~mm}$ below the interaural line. Coordinates were determined from the atlas of Paxinos and Watson (1998). Sham controls received injections of $0.4 \mu \mathrm{l}$ saline containing $0.01 \%$ ascorbic acid at the same coordinates. The infusion time was $4 \mathrm{~min}$, and the injector was left in place to allow diffusion for $5 \mathrm{~min}$. After surgery rats, received an injection of $2 \mathrm{ml}$ saline and an analgesic drug (Rimadyl; Pfizer, Karlsruhe, Germany; $4 \mathrm{mg} / \mathrm{kg}$, subcutaneously). Animals were allowed to recover for 5-11 days before training was started. We have no evidence that differences in recovery periods influenced individual behavioral performance.

Training and test procedure Training on the T-maze task was performed for 15 days, as described for Experiment 1 (Fig. 1). Upon completion of training, the choice behavior of sham controls and animals with AcbC DA depletion was examined in three testing blocks. In Testing Block 1, effortbased decision making was examined for 5 days under a restrictive feeding regimen, and in Testing Block 2 for 5 days under a free-feeding regimen. Upon completion of behavioral testing on Day 5 of Testing Block 1, the feeding regimen was shifted, and rats had ad libitum access to food on the subsequent days of Block 2. Testing on Day 1 of 
Block 2 started $18 \mathrm{~h}$ after ad libitum access to food had begun.

In Testing Blocks 1 and 2, a T-maze task was used as in Experiment 1; that is, rats could choose either to mount a barrier for HR in one goal arm or to obtain LR in the other goal arm without a barrier ("one barrier-condition"). This condition required a decision based on an integration of the effort and the reward magnitudes of the available response options.

On the subsequent Testing Block 3, decision making was examined under a restrictive feeding regimen for 3 days using a T-maze task variant in which identical barriers were present in the goal arms with both HR and LR ("two-barrier condition"). In the two-barrier condition, efforts were equated; hence, decision making was simply a function of reward magnitude. Importantly, the two-barrier condition allowed us to assess potential nonspecific lesion effects - for instance, sensorimotor impairments that interfered with barrier climbing. Upon completion of behavioral testing on Day 5 of Testing Block 2, the feeding regimen was shifted, and rats had restricted access to food once again. After four subsequent days under a restrictive feeding regimen, Testing Block 3 was run. Three days after completion of Testing Block 3, a consumption test was run to examine whether AcbC DA depletion altered the incentive value of the reward. Animals were placed in a separate cage containing a glass bowl filled with pellets. The animals had free access to the reward for $20 \mathrm{~min}$, and the amount consumed was measured by subtracting the weight of each glass bowl after the 20-min consumption test from the weight of the bowl before the test.

All rats completed every trial in all testing blocks. From all testing blocks, \%HR choices, RT (defined as the latency from insertion of an animal into the start arm until arrival at the reward position in the goal arm), and daily body weight were recorded.

Histology Tyrosine hydroxylase (TH) immunohistochemistry was used to assess the exact location and extent of the loss of DA terminals within the AcbC core. On completion of the behavioral testing, animals were euthanized by an overdose of isoflurane (Abbott, Wiesbaden, Germany) and perfused transcardially with $0.01 \%$ heparin sodium salt in phosphate-buffered saline (PBS), followed by $4 \%$ paraformaldehyde in PBS. The brains were removed, postfixed in paraformaldehyde for $24 \mathrm{~h}$, and dehydrated in $30 \%$ sucrose for at least $48 \mathrm{~h}$. Coronal brain sections were cut $(30 \mu \mathrm{m}$; Microm, Walldorf, Germany) in the region of the AcbC. The slices were initially washed in TRIS-buffered saline (TBS; $3 \times 10 \mathrm{~min}$ ), treated for $15 \mathrm{~min}$ with TBS containing $2 \%$ hydrogen peroxide and $10 \%$ methanol, washed again in TBS $(3 \times 10 \mathrm{~min})$ and then blocked for 20 min with $4 \%$ natural horse serum (NHS; Vector Laboratories, Burlingame, CA) in TBS containing $0.2 \%$ Triton X-100 (TBS-T; Sigma Aldrich). Slices were incubated overnight at $4^{\circ} \mathrm{C}$ in a primary antibody (mouse, anti-TH, 1:7,500 in TBS-T containing 4\% NHS; Immunostar, Hudson, WI), then washed in TBS-T $(3 \times 10 \mathrm{~min})$ and incubated in a secondary antibody (horse, antimouse, rat-adsorbed, biotinylated $\operatorname{IgG}[\mathrm{H}+\mathrm{L}], 1: 500$ in TBS-T containing $4 \%$ NHS; Vector Laboratories) for $90 \mathrm{~min}$ at room temperature. Using the biotin-avidin system, slices were washed in TBS-T containing the avidin-biotinylated enzyme complex (1:500, ABC-Elite Kit; Vector Laboratories) for $60 \mathrm{~min}$ at room temperature, washed in TBS $(3 \times 10 \mathrm{~min})$ and stained with 3,30-diaminobenzidine (DAB Substrate Kit, Vector Laboratories). The brain slices were then washed in TBS $(3 \times$ $10 \mathrm{~min}$ ), mounted on coated slides, dried overnight, dehydrated in ascending alcohol concentrations, treated with xylene, and finally coverslipped using DePex (Serva, Heidelberg, Germany). To determine the size and placement of the lesions, the TH immunoreactivity was analyzed under a microscope with reference to the atlas of Paxinos and Watson (1998).

Data analysis and statistics Choices of the HR arm, RTs, and body weights are given as percentage means \pm SEM. The data were subjected to a repeated measures ANOVA with two within-subjects factors (days of testing and testing blocks) and one between-subjects factor (treatment). All statistical computations were carried out with STATISTICA (Version 7.1; StatSoft, Inc., Tulsa, OK). The level of statistical significance ( $\alpha$ level) was set at $p \leq .05(\alpha$ levels $>.05$ were designated as n.s. [not significant]).

\section{Results}

\section{Experiment 1}

As is shown in Fig. 2, rats $(n=16)$ examined under a restrictive feeding regimen in Testing Block 1 displayed a strong preference for surmounting the barrier to obtain the large reward ( $\sim 95 \%$ HR preference), which became lower under a free-feeding regimen in Testing Block $2(\leq 80 \%$ mean HR preference). An ANOVA revealed significant main effects of testing block $[F(1,15)=17.52, p<.001]$ but not of day $[F(2,30)=1.82$, n.s.], and no testing block $\times$ day interaction $[F(2,30)=2.05$, n.s.]. Accordingly, RTs were shorter in Testing Block 1 as compared to Testing Block 2 (Fig. 3). An ANOVA revealed a significant main effect of testing block $[F(1,15)=29.93, p<.0001]$ but not of day $[F(2,30)=2.07$, n.s.], and no testing block $\times$ day interaction $[F(2,30)=1.56$, n.s.]. Furthermore, a shift from a restrictive to a free-feeding regimen resulted in a 


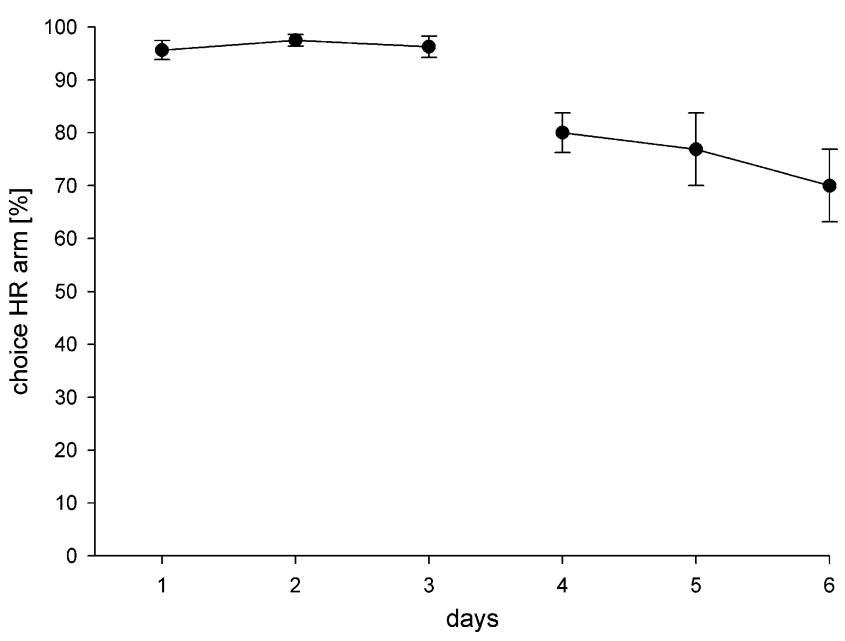

Fig. 2 Effects of a feeding regimen shift on effort-based decision making. Mean $( \pm S E M)$ percentages of high-reward (HR) arm choices per day are given. In Test Block 1 rats had restricted, and in Test Block 2 ad libitum, access to food. Each test block consisted of three consecutive test days. In both blocks, a 30-cm barrier was placed in the HR arm

pronounced body weight gain, as shown in Fig. 4. An ANOVA revealed significant main effects of testing block $[F(1,15)=838.01, p<.001]$ and day $[F(2,30)=7.72$, $p<.01]$ and a testing block $\times$ day interaction $[F(2,30)=$ $58.36, p<.001]$.

\section{Experiment 2}

Histology The lesion placements were assessed by reconstructing the damaged areas on standard stereotaxic atlas templates from Paxinos and Watson (1998). Figure 5a gives

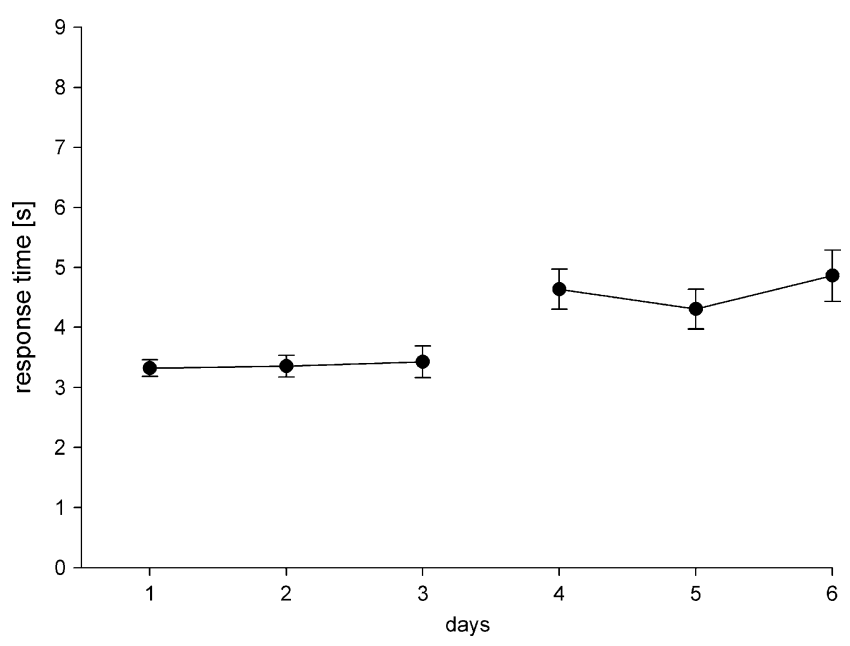

Fig. 3 Effects of a feeding regimen shift on response times (RTs). Mean $( \pm S E M)$ RTs per day are given. In Test Block 1 rats had restricted, and in Test Block 2 ad libitum, access to food. Each test block consisted of three consecutive test days. In both blocks, a $30-\mathrm{cm}$ barrier was placed in the HR arm

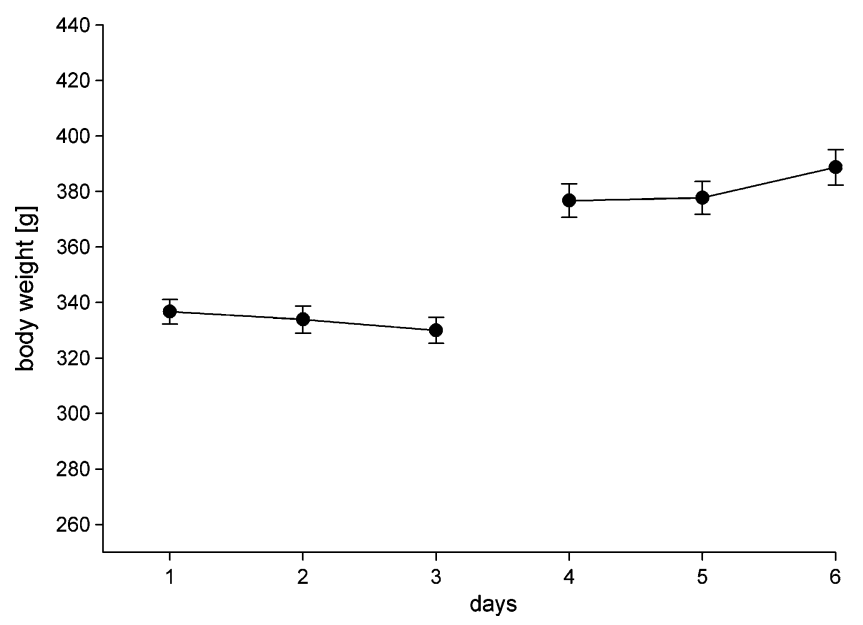

Fig. 4 Effects of a feeding regimen shift on body weights. Mean ( \pm $S E M$ ) body weights per day are given. In Test Block 1 rats had restricted, and in Test Block 2 ad libitum, access to food. Each test block consisted of three consecutive test days. In both blocks, a $30-\mathrm{cm}$ barrier was placed in the HR arm

a schematic illustration of the animals with the minimum and the maximum extents of AcbC lesion, respectively, as well as an animal with a representative AcbC lesion. A photomicrograph of a representative 6-OHDA-induced lesion is shown in Fig. 5b. TH-positive fibers in the AcbC were abundant in sham-lesioned rats $(n=14)$, but rare in rats with 6-OHDA lesions $(n=14)$. In most animals, the loss of TH-positive fibers in the AcbC appeared from about 2.2 to 0.2 relative to the bregma, with the maximum extension approximately at $1.5-1.2 \mathrm{~mm}$ relative to bregma. However, due to technical problems in TH immunohistochemistry, in a subset of lesioned rats reconstruction of the damaged areas was difficult. Therefore, 2 rats with ambiguous lesion placements were excluded. Furthermore, the rats with the smallest and largest lesions, respectively, given in Fig. 5a displayed an asymmetric (largest) or a partial (smallest) lesion of the AcbC. We cannot exclude the possibility that problems with $\mathrm{TH}$ immunohistochemistry may have obscured a more widespread loss of TH fibers in the less-affected side of the AcbC in the animal with the asymmetric large lesion. In line with this idea, behavioral results revealed that choice behavior in this animal was markedly altered. Likewise, problems with $\mathrm{TH}$ immunohistochemistry may have masked a more widespread loss of TH fibers in the animal with the smallest lesion-for instance, in the more anterior parts of the AcbC. However, the behavioral data indicated that choice behavior was least affected in this out of all lesioned animals, pointing to a relatively small AcbC DA depletion in this subject. Importantly, other lesioned animals displayed typical AcbC lesions (Fig. 5a); in these animals, we found no evidence for a relation between lesion size or location and the magnitude of behavioral effects. Furthermore, in these 
Fig. 5 (a) Loss of TH-positive fibers in 6-hydroxydopamine(6-OHDA-)lesioned animals in Experiment 2. The drawings show a reconstruction of the regions that were nearly devoid of TH-immunoreactive fibers, indicating in each slice the animal with the largest (unilateral) lesion (grey areas) and the animal with the smallest lesion (black areas). An animal with a representative lesion is also shown (cross-hatched areas). The numbers indicate the distances from bregma, in millimeters. (b) Representative photomicrographs of a 6OHDA-induced loss of THimmunoreactive fibers in the AcbC (top: AcbC sham lesion; bottom: AcbC DA depletion) a

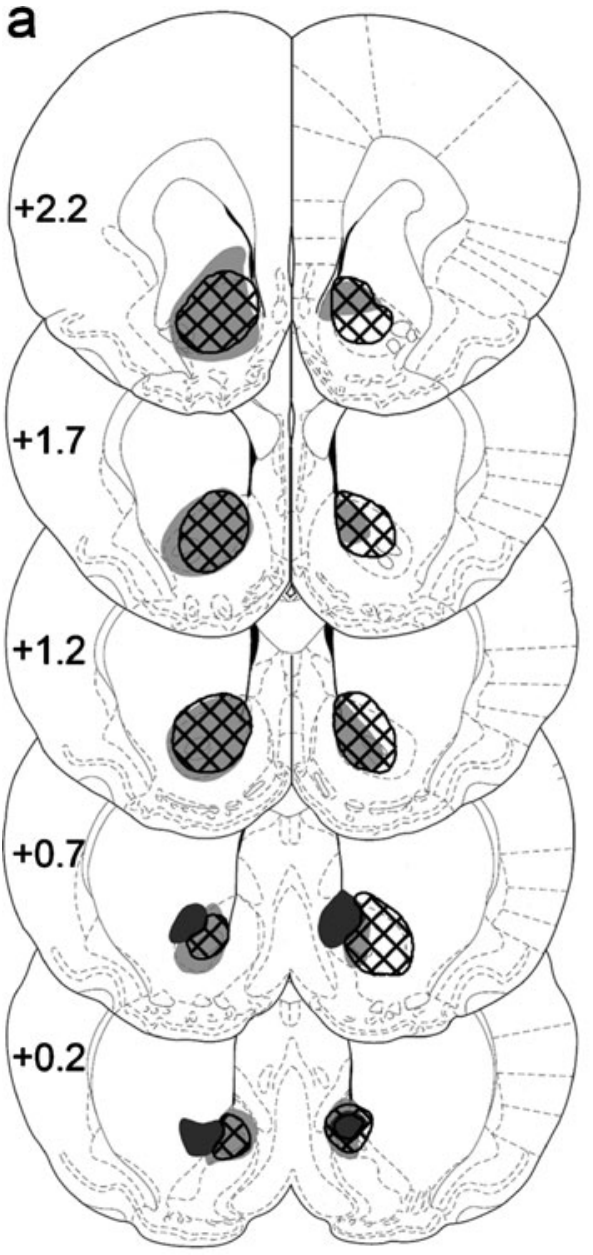

b

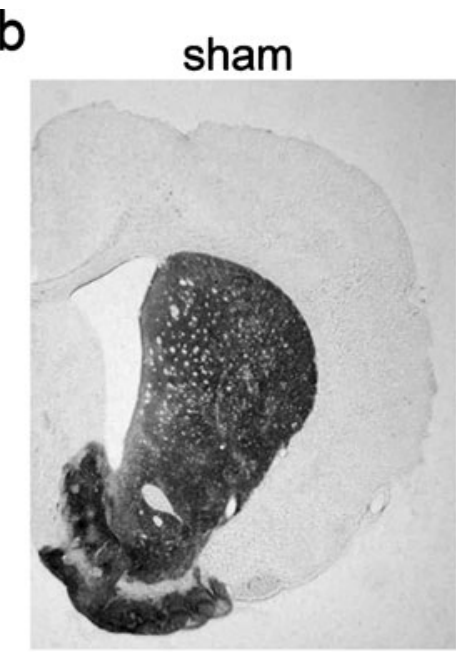

AcbC lesion

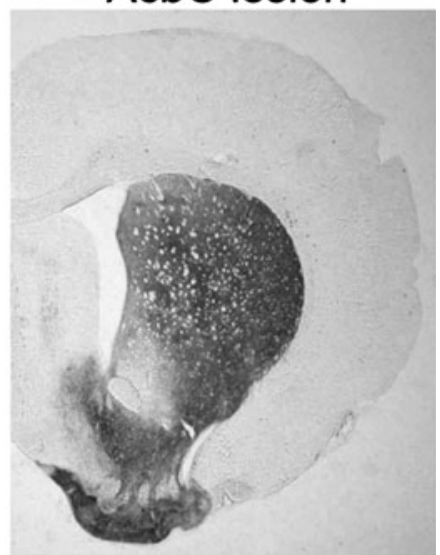

animals, the location and size of AcbC lesions largely corresponded to those in an earlier study using an almost identical protocol (Lex \& Hauber, 2010). Rather than employing TH immunohistochemistry to assess the exact location and size of 6-OHDA-induced loss of striatal DA fibers, as used here, many behavioral studies have measured the degree of DA depletion in striatal tissue homogenates (e.g., Hauber, Bubser, \& Schmidt, 1994; Salamone et al., 1991). Therefore, relatively little is known about the behavioral effects of AcbC DA depletion as a function of lesion size and location. Previous studies had reported that intrastriatal infusion of solutions with the same concentrations of 6-OHDA used here $(4 \mu \mathrm{g} / \mu \mathrm{l})$ profoundly reduced tissue concentrations of DA (80\%) (e.g., Kelly, Seviour, \& Iversen, 1975; Winn \& Robbins, 1985). Likewise, intrastriatal infusion of solutions containing 4 $7 \mu \mathrm{g} / \mu \mathrm{l}$ 6-OHDA markedly reduced TH-positive fiber density (Chapman \& Zahm, 1996; Weissenborn \& Winn, 1992). Together, these findings provide support for our observation that rats subjected to 6-OHDA infusions had near-complete AcbC DA depletion.
Training On training days without a barrier, sham controls and rats with AcbC DA depletion both had a higher preference for obtaining the large reward (Fig. 6). An ANOVA revealed no effects of treatment $[F(1,26)=$ 0.11 , n.s.] but a significant main effect of days $[F(2,52)=$ $9.39, p<.001]$, and no days $\times$ treatment interaction $[F(2$, $52)=0.39$, n.s.].

During barrier training, sham controls displayed a preference for surmounting the barrier to obtain the large reward that was increasingly higher than that of the rats with AcbC DA depletion (Fig. 6). An ANOVA showed significant main effects of testing block $[F(2,52)=5.57$, $p<.01]$ and days $[F(2,52)=16.71, p<.001]$, as well as a trend for an effect of treatment $[F(1,26)=3.28, p=.08, \mathrm{n}$. s.]. Furthermore, there was no overall testing block $x$ treatment interaction $[F(2,52)=1.04$, n.s.]. For a more detailed analysis, we examined choice behavior in the last training block separately. An ANOVA revealed a significant main effect of treatment $[F(1,26)=5.05, p<.05]$, but no main effect of days $[F(2,52)=0.28$, n.s. $]$ and no days $\times$ treatment interaction $[F(2,52)=0.40$, n.s. $]$. 


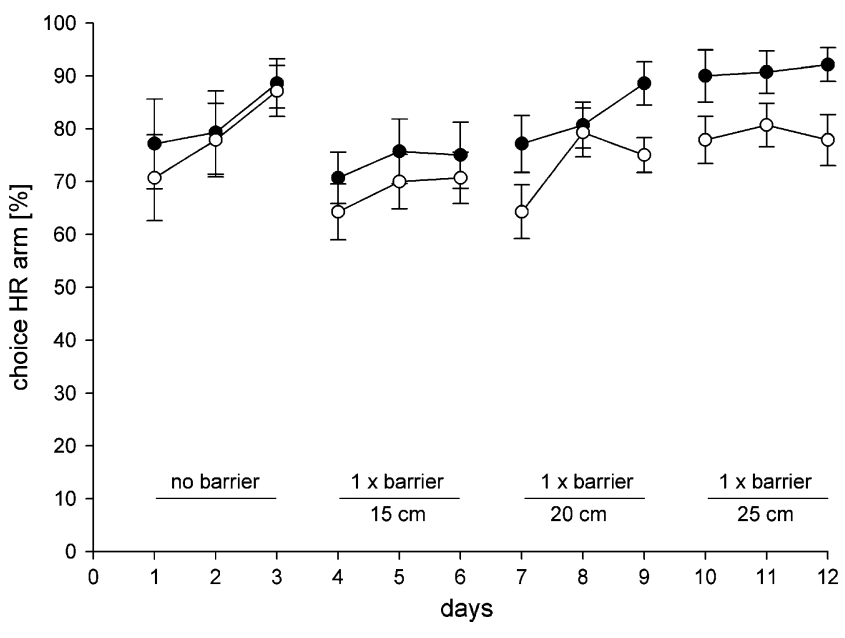

Fig. 6 Training on the effort-based decision-making task. Mean $( \pm$ $S E M$ ) percentages of high-reward (HR) arm choices per day in shamlesioned ( $n=14$, filled circles) and 6-hydroxydopamine-lesioned $(n=$ 14 , open circles) rats are given. In the first block, no barrier was present, and in subsequent blocks, barriers of increasing sizes were introduced in the HR arm

Choice behavior First, decision making was examined in the one-barrier condition; that is, rats could choose either to mount a barrier for HR in one goal arm or to obtain LR in the other goal arm without a barrier. Under a restrictive feeding regimen, sham controls displayed a higher preference for surmounting the barrier to obtain the large reward than did rats with AcbC DA depletion (Fig. 7). A shift from

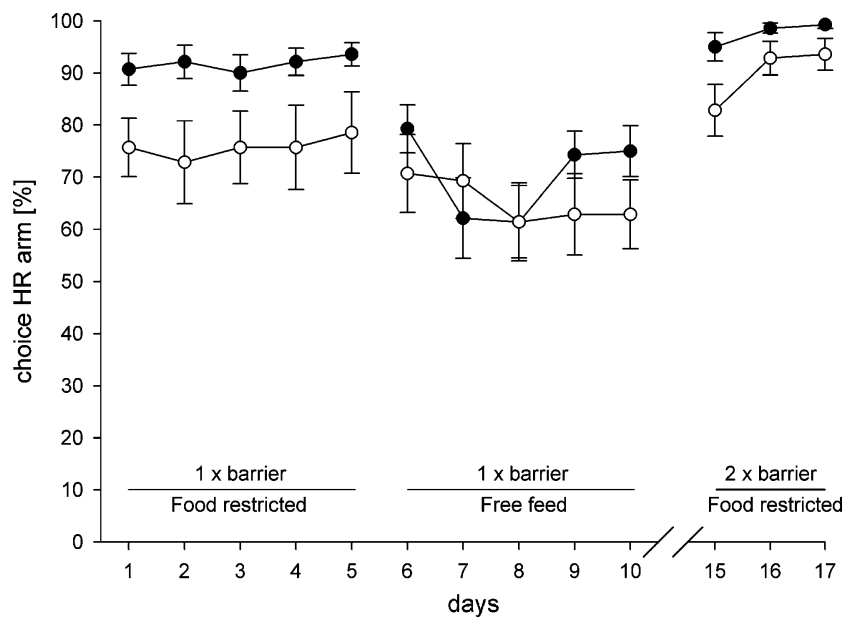

Fig. 7 Effects of 6-hydroxydopamine (6-OHDA) lesions of the AcbC on effort-based decision making. Mean $( \pm S E M)$ percentages of highreward (HR) arm choices per day in sham-lesioned $(n=14$, filled circles) and 6-OHDA-lesioned ( $n=14$, open circles) rats are given. In Test Blocks 1 and 3 rats had restricted, and in Test Block 2 ad libitum, access to food. Test Blocks 1 and 2 consisted of five consecutive test days. In Blocks 1 and 2, a 30-cm barrier was placed in the HR arm (one-barrier condition). Test Block 3 consisted of three consecutive test days with identical $30-\mathrm{cm}$ barriers placed in each goal arm (twobarrier condition) a restricted to a free-feeding regimen reduced in both treatment groups the HR arm preference. However, over days, HR arm preference in the sham controls became higher relative to that of rats with AcbC DA depletion. An ANOVA revealed significant main effects of testing block $[F(1,26)=18.18, p<.001]$ and days $[F(4,104)=3.49$, $p<.05]$, but no main effect of treatment $[F(1,26)=2.35$, n. s.]. Furthermore, there was no overall testing block $\times$ treatment interaction $[F(1,26)=2.21$, n.s.], but there was a near-significant block $\times$ days interaction $[F(4,104)=2.36$, $p=.06$, n.s. $]$ as well as a near-significant testing block $\times$ days $\times$ treatment interaction $[F(4,104)=2.38, p=.06$, n. s.]. Furthermore, additional ANOVAs were run on the data from each testing block separately. In the first block, there was a significant main effect of treatment $[F(1,26)=5.08$, $p<.05]$ but no main effect of days $[F(4,104)=0.52$, n.s. $]$ and no treatment $\times$ days interaction $[F(4,104)=0.26$, n.s. $]$. By contrast, in the second testing block, there was no main effect of treatment $[F(1,26)=0.35$, n.s. $]$, but a main effect of days did appear $[F(4,104)=4.61, p<.01]$, as well as a treatment $\times$ days interaction $[F(4,104)=3.24, p<.05]$. For a more detailed analysis, a separate ANOVA for each treatment group was run on HR preferences in Testing Blocks 1 versus 2. The results showed that HR arm preference was lower in Testing Block 2 relative to Testing Block 1 both in sham controls [main effect of block: $F(1,13)=15.08$, $p<.01]$ and, to a less pronounced extent, in lesioned rats [main effect of block: $F(1,13)=4.27, p=.06$, n.s.].

Subsequently, after a shift to a restricted feeding regimen, decision making was tested in the two-barrier condition-that is, the HR and LR goal arms were both blocked by a barrier. In this testing block, HR arm preferences became markedly higher relative to the preceding testing blocks both in sham controls and, albeit by a less pronounced amount, in rats with AcbC DA depletion. An ANOVA revealed significant main effects of treatment $[F(1,26)=5.17, p<.05]$ and days $[F(2,52)=7.84$, $p<.01]$, but no treatment $\times$ days interaction $[F(2,52)=$ 1.58 , n.s.].

Response times The results showed that, in the one-barrier condition, RTs were influenced by feeding regimen and treatment (Fig. 8). An ANOVA revealed a significant effect of testing block $[F(1,26)=5.30, p<.05]$ but not of days $[F$ $(4,104)=0.82$, n.s.], along with no effect of treatment $[F$ $(1,26)=1.05$, n.s.] and no interactions between these factors $(F>0.66$, n.s.). For a more detailed analysis, an ANOVA was run on the data from each testing block separately. In the first testing block, there was a significant main effect of treatment $[F(1,26)=4.20, p=.05]$, a significant main effect of days $[F(4,104)=7.75, p<.001]$, and no treatment $\times$ days interaction $[F(4,104)=1.11$, n.s. $]$. By contrast, in the second testing block, there was no main 


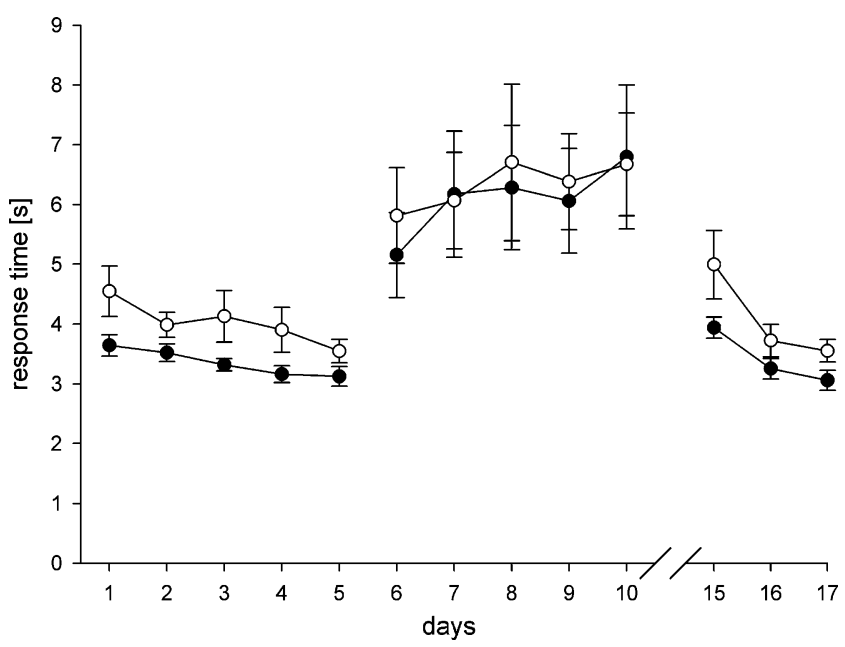

Fig. 8 Effects of 6-hydroxydopamine (6-OHDA) lesions of the AcbC on response times (RTs). Mean $( \pm S E M)$ RTs per day in sham-lesioned ( $n=14$, filled circles) and 6-OHDA-lesioned ( $n=14$, open circles) rats are given. In Test Blocks 1 and 3 rats had restricted, and in Test Block 2 ad libitum, access to food. Test Blocks 1 and 2 consisted of five consecutive test days. In Blocks 1 and 2, a 30-cm barrier was placed in the HR arm (one-barrier condition). Test Block 3 consisted of three consecutive test days with identical $30-\mathrm{cm}$ barriers placed in each goal arm (two-barrier condition)

effect of treatment $[F(1,26)=0.86$, n.s. $]$ or of days $[F(4$, $104)=0.88$, n.s. $]$, and no treatment $\times$ days interaction $[F(4$, $104)=1.17$, n.s.].

After a shift to a restricted feeding regimen, animals were tested in the two-barrier condition. In this third testing block, animals became markedly faster relative to the preceding testing block. Furthermore, sham controls displayed shorter RTs to obtain food reward than did rats with AcbC DA depletion. An ANOVA revealed a nearsignificant effect of treatment $[F(1,26)=3.53, p=.07, \mathrm{n}$. s.]. Furthermore, there was a significant main effect of days $[F(2,52)=22.20, p<.001]$, but no treatment $\times$ days interaction $[F(2,52)=0.76$, n.s.].

Body weights At time of surgery, the body weights of the sham controls and the rats with DA AcbC depletion did not differ [sham controls, $320.3 \pm 19.6 \mathrm{~g}$; lesioned rats, $321.0 \pm$ $19.6 \mathrm{~g} ; t(26)=0.03$, n.s.]. The results demonstrate that, during testing in the one-barrier condition, body weights were markedly influenced by the testing block and, albeit in a less pronounced manner, by treatment (Fig. 9). An ANOVA revealed significant effects of testing block $[F(1$, $26)=930.02, p<.001]$ and days $[F(4,104)=59.70$, $p<.001]$, but no effect of treatment $[F(1,26)=0.72$, n.s.]. Furthermore, there was a testing block $\times$ days interaction $[F$ $(4,104)=40.74, p<.001]$, but no testing block $\times$ treatment interaction $[F(1,26)=0.11$, n.s. $]$ and no testing block $\times$ days $\times$ treatment interaction $[F(4,104)=0.17$, n.s $]$. For a more detailed analysis, an additional ANOVA was run on

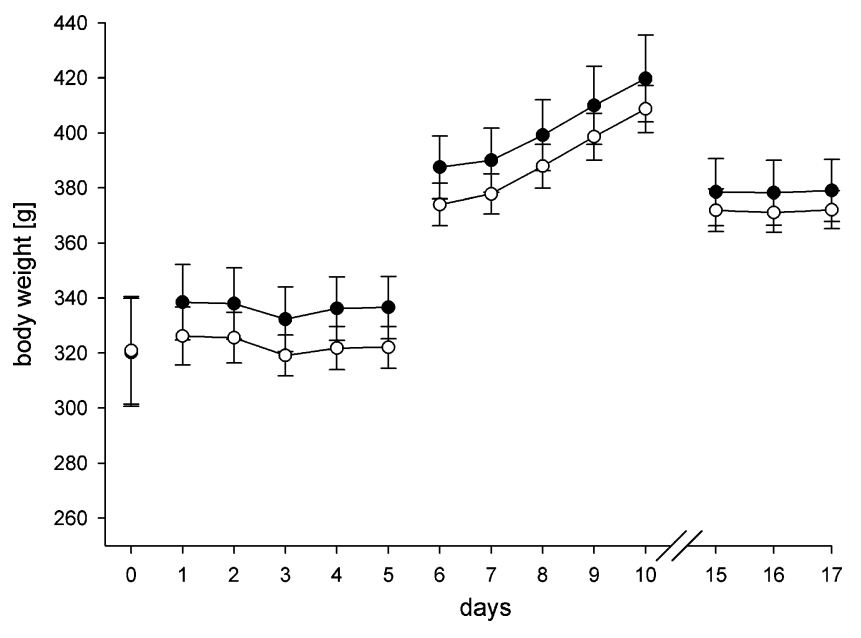

Fig. 9 Effects of 6-hydroxydopamine (6-OHDA) lesions of the AcbC on body weights. Mean $( \pm S E M)$ body weights per day in shamlesioned ( $n=14$, filled circles) and 6-OHDA-lesioned ( $n=14$, open circles) rats are given. In Test Blocks 1 and 3 rats had restricted, and in Test Block 2 ad libitum, access to food. Test Blocks 1 and 2 consisted of five consecutive test days. In Blocks 1 and 2, a 30-cm barrier was placed in the HR arm (one-barrier condition). Test Block 3 consisted of three consecutive test days with identical $30-\mathrm{cm}$ barriers placed in each goal arm (two-barrier condition)

the data from each testing block separately. In the first testing block, there was a significant main effect of days $[F$ $(4,104)=3.65, p<.01]$ but no main effect of treatment $[F$ $(1,26)=0.82$, n.s. $]$ and no treatment $\times$ days interaction $[F$ $(4,104)=0.14$, n.s.]. Similarly, in the second testing block, there was a significant main effect of days $[F(4,104)=$ $81.04, p<.001]$ but no main effect of treatment $[F(1,26)=$ 0.61 , n.s. $]$ and no treatment $\times$ days interaction $[F(4,104)=$ 0.11 , n.s.].

Subsequently, after a shift to a restricted feeding regimen, rats of both treatment groups continued to show stable body weights. An ANOVA revealed no main effect of treatment $[F(1,26)=0.25$, n.s. $]$ or of days $[F(2,52)=$ 0.49 , n.s. $]$, and there was no treatment $\times$ days interaction $[F$ $(2,52)=0.06$, n.s.].

Consumption test The consumption test revealed no differences in the amounts of pellets eaten among sham controls and 6-OHDA-lesioned rats (sham controls, $16.1 \pm 0.9 \mathrm{~g}$; lesioned rats, $16.0 \pm 0.6 \mathrm{~g} ; t(26)=0.13$, n.s.].

\section{Discussion}

Experiment 1 revealed that effort-based decision making in intact rats was influenced by the motivational state; that is, a shift from a hungry to a sated state reduced their preference for the high-effort-HR option when they had the choice to obtain a lower reward with little effort. 
Experiment 2 demonstrated that, relative to sham controls, rats with AcbC DA depletion had a reduced preference to effortful but HR actions in both hungry and sated states. Importantly, the results further revealed that effort-based decision making in the lesioned rats, as in the sham controls, was still sensitive to a shift from a hungry to a sated state- that is, their preferences for effortful HR actions became lower after shift from a restricted to a free-feeding regimen. In other words, in rats with AcbC DA depletion, the preference for the high-effort-HR option was generally lower, but a shift in motivational state could still influence the allocation of effort they devoted to obtaining rewards. These findings suggest that AcbC DA may not necessarily be involved in mediating the effects of motivational states on decision making.

Not unexpectedly, the results of Experiment 1 demonstrated that in intact rats a motivational shift from a hungry to a sated state influenced effort-based decision making in a T-maze task; that is, in the sated state, the number of effortful HR arm choices became lower, and RTs became longer. These findings suggest that effortrelated decision-making policies are variable and subject to modulation by changing motivational states. In line with this notion, satiety manipulations in operant effortbased decision-making tasks markedly decreased HR lever preference and increased response latencies (Endepols et al., 2010; Floresco et al. 2008; Salamone et al., 1991). Likewise, St. Onge and Floresco (2009) recently revealed that riskrelated decision-making policies are modulated by shifts in motivational state.

Experiment 2 showed that, if tested in a hungry state in the one barrier-condition, rats with AcbC DA depletion, unlike sham controls, displayed a reduced HR arm preference. Furthermore, if tested in a hungry state in the two-barrier condition - that is, if the efforts were equated for the two response options - the preference was increased for the HR response option relative to the one-barrier condition in both sham controls and rats with AcbC DA depletion. Thus, rats adapted their choice behavior when cost-benefit ratios of response options in the one- versus two-barrier conditions changed, suggesting that they made cost-benefit-related decisions and did not respond in a habit-like manner. However, in the two-barrier condition, HR arm preference was significantly lower in rats with AcbC DA depletion than in sham controls. Thus, the altered decision making seen in the one-barrier condition could be interpreted as reflecting lesion-induced motor or spatial impairments, or as a result of problems in reward magnitude discrimination or reward valuation. Yet this possibility seems unlikely, because rats with AcbC DA depletion still attained a high HR arm preference and differed from sham controls predominantly in terms of their higher interindividual variance. Furthermore, the consump- tion test revealed that AcbC DA depletion did not affect the palatability of food reward. Also, since the time required to surmount the barrier in our task was short $(<1.5 \mathrm{~s})$, differential delays to obtain HR versus LR might not bias responding in the one-barrier situation. Taken together, our present findings in hungry rats provide further support to the notion that AcbC DA depletions shifted the preference from high-value-high-cost options to low-value-low-cost alternatives (Cousins et al., 1996; Salamone et al., 2007; Salamone et al., 1994). Furthermore, our data are consistent with recent neurochemical and electrophysiological studies indicating a key role of the nucleus accumbens in costbenefit decisions. For instance, in rats making effort-related decisions, accumbens DA signals convey important information on expected rewards (Wanat, Kuhnen, \& Phillips, 2010), while accumbens neurons encode predicted and ongoing reward costs (Day, Jones, \& Carelli, 2011).

Relative to lesioned rats, sham controls in the sated state displayed an increasing HR arm preference on Testing Days 4 and 5 that, nevertheless, remained on a lower overall level than their preferences in a food-restricted state. These findings are consistent with the idea that effort-related decision-making policies are subject to modulation by motivational states, but they also suggest that in intact rats this modulation is particularly pronounced immediately after a shift in motivational state. In line with this notion, Floresco et al. (2008) observed in intact rats tested in an operant effort-related task that a shift from a restricted to a free-feeding regimen had prominent immediate effects on choice behavior - that is, the shift reduced the preference for the high-value-high-cost option on the first testing day, an effect that disappeared over four subsequent days with ad libitum access to food.

The observation that in rats with AcbC DA depletion HR arm preference was reduced in the hungry state and, by a less pronounced amount, in the sated state suggests that AcbC DA is critically involved in regulating how much effort to invest for reward, regardless of motivational state. Consistent with numerous earlier studies (Cousins et al., 1996; Salamone, 1994; Salamone, Kurth, McCullough, Sokolowski, \& Cousins, 1993; Taghzouti, Louilot, Herman, Le Moal, \& Simon, 1985; Weissenborn \& Winn, 1992; Winn \& Robbins, 1985), our results further revealed that a shift from a hungry to a sated state markedly slowed response execution and increased body weights in sham controls and, to a lesser extent, in rats with AcbC DA depletion.

Furthermore, our results demonstrated that effort-related decision making in rats with AcbC DA depletion, as in sham controls, was still sensitive to a shift in motivational state; that is, in lesioned rats in either a hungry or a sated state, preferences for effortful large-reward actions were lower, but this lowered preference was reduced further by a shift from a restricted to a free-feeding regimen. Of note, after the motivational downshift, HR arm preferences 
became rapidly lower in sham controls, but only gradually lower in lesioned rats. Thus, it is possible that, although animals in both treatment groups were sensitive to the motivational downshift, the learning mechanisms that underlie the behavioral manifestations of motivational changes (e.g., Niv, Joel, \& Dayan, 2006) could be distinct in sham controls and lesioned animals.

It is well known that decision-making policies are subject to modulation by motivational states (Bindra, 1969, 1974). A number of studies have implicated DA transmission, in particular in the nucleus accumbens, in mediating the effects of motivational states on behavior (Willner, Chawla, Sampson, Sophokleous, \& Muscat, 1988). For instance, microdialysis studies have demonstrated that during feeding, accumbens DA release was lower in sated than in hungry rats (Ahn \& Phillips, 1999; Bassareo \& Di Chiara, 1999; Wilson et al., 1995). However, our present findings imply that AcbC DA transmission is not necessarily involved in mediating the effects of motivational states on behavior. In line with this account, the effects of AcbC DA depletions tested in a concurrent leverpress/lab chow feeding choice task differed markedly from the effects of motivational downshifts due to prefeeding (Salamone et al., 1991) or administration of appetite suppressant drugs (Cousins \& Salamone, 1994; Salamone \& Correa, 2002; Sink, Vemuri, Olszewska, Makriyannis, \& Salamone, 2008, see Salamone et al., 2007, for review).

It is conceivable that in rats with AcbC DA depletion, other target regions of DA signals might mediate the effects of a shift in motivational state on effort allocation. For instance, microdialysis in rats tested in an operant task revealed that tonic DA conveys information about the costs and benefits of instrumental responding to various striatal subregions. Importantly, during leverpressing for food, the increase in striatal DA release was attenuated when rats were sated before testing (Ostlund, Wassum, Murphy, Balleine, \& Maidment, 2011). The authors also detected that, among several striatal subregions investigated, only in the AcbS did the suppressive effects of satiety on DA efflux vary with the effect of satiety on leverpressing. These findings suggest that in a cost-benefit task such as the one used in our study, the AcbS could be one important subregion that, via DA-ergic mechanisms, mediates the effects of a downshift in motivation on effort allocation. Contrasting with this view, a recent study suggested that the AcbC rather than the AcbS is part of the neural circuit mediating effort-based decision making (Ghods-Sharifi \& Floresco, 2010). However, to allow for an adaption to motivational states, the AcbS could impact in an indirect manner on the neural circuit mediating effort-based decision making - for instance, by feedforward propagation of information from the AcbS to the AcbC via striatonigrostriatal pathways (Haber, Fudge, \& McFarland, 2000).
Furthermore, other target areas of DA fibers, such as prefrontal cortex and basolateral amygdala, also play critical roles in tracking changes in motivational states (Bassareo \& Di Chiara, 1999; Moscarello, Ben-Shahar, \& Ettenberg, 2007, 2009). Thus, further studies will be needed to delineate the role of DA in these brain areas in mediating the effects of motivational shifts on effort-based decision making. Also, apart from DA, other neurochemical systems, such as opioid systems (Wassum, Ostlund, Maidment, \& Balleine, 2009), could contribute toward mediating the effects of motivational shifts on decision making.

\section{Conclusions}

Consistent with earlier studies (Salamone et al., 2007), our present results confirmed a critical role for AcbC DA in effort-related decision making. Importantly, our findings further suggest that AcbC DA is not necessarily involved in mediating the effects of a shift in motivational state on decision-making policies.

Author note This research was supported by DFG Grant HA2340/ 9-1.

\section{References}

Ahn, S., \& Phillips, A. G. (1999). Dopaminergic correlates of sensoryspecific satiety in the medial prefrontal cortex and nucleus accumbens of the rat. Journal of Neuroscience, 19, RC29.

Bassareo, V., \& Di Chiara, G. (1999). Differential responsiveness of dopamine transmission to food-stimuli in nucleus accumbens shell/core compartments. Neuroscience, 89, 637-641.

Bindra, D. (1969). A unfied interpretation of emotion and motivation. Annals of the New York Academy of Sciences, 159, 1071-1083.

Bindra, D. (1974). A motivational view of learning, performance, and behavior modification. Psychology Review, 81, 199-213.

Chapman, M. A., \& Zahm, D. S. (1996). Altered Fos-like immunoreactivity in terminal regions of the mesotelencephalic dopamine system is associated with reappearance of tyrosine hydroxylase immunoreactivity at the sites of focal 6-hydroxydopamine lesions in the nucleus accumbens. [Research Support, U.S. Gov't, P.H. S.]. Brain Research, 736, 270-279.

Cousins, M. S., Atherton, A., Turner, L., \& Salamone, J. D. (1996). Nucleus accumbens dopamine depletions alter relative response allocation in a T-maze cost/benefit task. Behavior Brain Research, 74, 189-197.

Cousins, M. S., \& Salamone, J. D. (1994). Nucleus accumbens dopamine depletions in rats affect relative response allocation in a novel cost/benefit procedure. Pharmacolog and Biochem Behavior, 49, 85-91.

Day, J. J., Jones, J. L., \& Carelli, R. M. (2011). Nucleus accumbens neurons encode predicted and ongoing reward costs in rats. Eur Journal of Neuroscience, 33, 308-321. doi:10.1111/j.1460-9568.2010.07531.x

Endepols, H., Sommer, S., Backes, H., Wiedermann, D., Graf, R., \& Hauber, W. (2010). Effort-based decision making in the rat: An [18 F]fluorodeoxyglucose micro positron emission tomography study. Journal of Neuroscience, 30, 9708-9714. doi:10.1523/ JNEUROSCI.1202-10.2010 
Floresco, S. B., Tse, M. T., \& Ghods-Sharifi, S. (2008). Dopaminergic and glutamatergic regulation of effort- and delay-based decision making. Neuropsychopharmacology, 33, 1966-1979. doi:10.1038/sj.npp.1301565

Ghods-Sharifi, S., \& Floresco, S. B. (2010). Differential effects on effort discounting induced by inactivations of the nucleus accumbens core or shell. Behavioral Neuroscience, 124, 179191. doi: $10.1037 / \mathrm{a} 0018932$

Haber, S. N., Fudge, J. L., \& McFarland, N. R. (2000). Striatonigrostriatal pathways in primates form an ascending spiral from the shell to the dorsolateral striatum. Journal of Neuroscience, 20, 2369-2382.

Hauber, W., Bubser, M., \& Schmidt, W. J. (1994). 6Hydroxydopamine lesion of the rat prefrontal cortex impairs motor initiation but not motor execution. [Research Support, Non-U.S. Gov't]. Experimental Brain Research, 99, 524-528.

Hauber, W., \& Sommer, S. (2009). Prefrontostriatal circuitry regulates effort-related decision making. Cerebral Cortex, 19, 2240-2247. doi:10.1093/cercor/bhn241

Kelly, P. H., Seviour, P. W., \& Iversen, S. D. (1975). Amphetamine and apomorphine responses in the rat following 6-OHDA lesions of the nucleus accumbens septi and corpus striatum. Brain Research, 94, 507-522.

Lex, B., \& Hauber, W. (2010). The role of nucleus accumbens dopamine in outcome encoding in instrumental and Pavlovian conditioning. Neurobiology of Learning and Memory, 93, 283290. doi:10.1016/j.nlm.2009.11.002

Moscarello, J. M., Ben-Shahar, O., \& Ettenberg, A. (2007). Dynamic interaction between medial prefrontal cortex and nucleus accumbens as a function of both motivational state and reinforcer magnitude: a c-Fos immunocytochemistry study. Brain Research, 1169, 69-76.

Moscarello, J. M., Ben-Shahar, O., \& Ettenberg, A. (2009). Effects of food deprivation on goal-directed behavior, spontaneous locomotion, and c-Fos immunoreactivity in the amygdala. Behavioural Brain Research, 197, 9-15. doi:10.1016/j.bbr.2008.07.025

Niv, Y., Joel, D., \& Dayan, P. (2006). A normative perspective on motivation [Research Support, Non-U.S. Gov't Review]. Trends in Cognitive Sciences, 10, 375-381. doi:10.1016/j.tics.2006.06.010

Ostlund, S. B., Wassum, K. M., Murphy, N. P., Balleine, B. W., \& Maidment, N. T. (2011). Extracellular dopamine levels in striatal subregions track shifts in motivation and response cost during instrumental conditioning. Journal of Neuroscience, 31, 200207. doi:10.1523/JNEUROSCI.4759-10.2011

Paxinos, G., \& Watson, C. (1998). The rat brain in stereotaxic coordinates (4th ed.). San Diego: Academic Press.

Phillips, P. E., Walton, M. E., \& Jhou, T. C. (2007). Calculating utility: preclinical evidence for cost-benefit analysis by mesolimbic dopamine. Psychopharmacology, 191, 483-495. doi:10.1007/ s00213-006-0626-6

Salamone, J. D. (1994). The involvement of nucleus accumbens dopamine in appetitive and aversive motivation. Behavioural Brain Research, 61, 117-133.

Salamone, J. D., \& Correa, M. (2002). Motivational views of reinforcement: Implications for understanding the behavioral functions of nucleus accumbens dopamine [Research Support, U.S. Gov't, Non-P. H.S. Review]. Behavioural Brain Research, 137, 3-25.

Salamone, J. D., Correa, M., Farrar, A., \& Mingote, S. M. (2007). Effort-related functions of nucleus accumbens dopamine and associated forebrain circuits. Psychopharmacology, 191, 461482. doi:10.1007/s00213-006-0668-9

Salamone, J. D., Cousins, M. S., \& Bucher, S. (1994). Anhedonia or anergia? Effects of haloperidol and nucleus accumbens dopamine depletion on instrumental response selection in a T-maze cost/ benefit procedure. Behavioural Brain Research, 65, 221-229.

Salamone, J. D., Kurth, P. A., McCullough, L. D., Sokolowski, J. D., \& Cousins, M. S. (1993). The role of brain dopamine in response initiation: Effects of haloperidol and regionally specific dopamine depletions on the local rate of instrumental responding. Brain Research, 628, 218-226.

Salamone, J. D., Steinpreis, R. E., McCullough, L. D., Smith, P., Grebel, D., \& Mahan, K. (1991). Haloperidol and nucleus accumbens dopamine depletion suppress lever pressing for food but increase free food consumption in a novel food choice procedure. Psychopharmacology, 104, 515-521.

Schweimer, J., \& Hauber, W. (2005). Involvement of the rat anterior cingulate cortex in control of instrumental responses guided by reward expectancy. Learning and Memory, 12, 334-342. doi: $10.1101 / \mathrm{lm} .90605$

Sink, K. S., Vemuri, V. K., Olszewska, T., Makriyannis, A., \& Salamone, J. D. (2008). Cannabinoid CB1 antagonists and dopamine antagonists produce different effects on a task involving response allocation and effort-related choice in foodseeking behavior. [Comparative Study Research Support, N.I.H., extramural]. Psychopharmacology, 196, 565-574. doi:10.1007/ s00213-007-0988-4

St. Onge, J. R., \& Floresco, S. B. (2009). Dopaminergic modulation of risk-based decision making. [Research Support, Non-U.S. Gov't]. Neuropsychopharmacology, 34, 681-697. doi:10.1038/ npp. 2008.121

Taghzouti, K., Louilot, A., Herman, J. P., Le Moal, M., \& Simon, H. (1985). Alternation behavior, spatial discrimination, and reversal disturbances following 6-hydroxydopamine lesions in the nucleus accumbens of the rat. Behavioral and Neural Biology, 44, 354-363.

Viana, D. S., Gordo, I., Sucena, E., \& Moita, M. A. (2010). Cognitive and motivational requirements for the emergence of cooperation in a rat social game. PLoS One, 5, e8483. doi:10.1371/journal. pone. 0008483

Walton, M. E., Bannerman, D. M., Alterescu, K., \& Rushworth, M. F. (2003). Functional specialization within medial frontal cortex of the anterior cingulate for evaluating effort-related decisions. Journal of Neuroscience, 23, 6475-6479.

Wanat, M. J., Kuhnen, C. M., \& Phillips, P. E. (2010). Delays conferred by escalating costs modulate dopamine release to rewards but not their predictors. Journal of Neuroscience, 30, 12020-12027. doi:10.1523/JNEUROSCI.2691-10.2010

Wassum, K. M., Ostlund, S. B., Maidment, N. T., \& Balleine, B. W. (2009). Distinct opioid circuits determine the palatability and the desirability of rewarding events. Proceedings of the National Academy of Sciences, 106, 12512-12517. doi:10.1073/ pnas.0905874106

Weissenborn, R., \& Winn, P. (1992). Regulatory behaviour, exploration and locomotion following NMDA or 6-OHDA lesions in the rat nucleus accumbens. Behavioural Brain Research, 51, 127-137.

Willner, P., Chawla, K., Sampson, D., Sophokleous, S., \& Muscat, R. (1988). Tests of functional equivalence between pimozide pretreatment, extinction and free feeding. Psychopharmacology, 95, 423-426.

Wilson, C., Nomikos, G. G., Collu, M., \& Fibiger, H. C. (1995). Dopaminergic correlates of motivated behavior: Importance of drive. Journal of Neuroscience, 15, 5169-5178.

Winn, P., \& Robbins, T. W. (1985). Comparative effects of infusions of 6hydroxydopamine into nucleus accumbens and anterolateral hypothalamus induced by 6-hydroxydopamine on the response to dopamine agonists, body weight, locomotor activity and measures of exploration in the rat. Neuropharmacology, 24, 25-31. 